\title{
Estresse em crianças e adolescentes com Síndrome de Williams-Beuren em idade escolar
}

\author{
Vera Alice Alcantara dos Santos Amaral \\ Michele Moreira Nunes \\ Rachel Sayuri Honjo \\ Roberta Lelis Dutra \\ Francisco Baptista Assumpção Jr \\ Chong Ae Kim
}

\begin{abstract}
Resumo
Síndrome de Williams-Beuren é uma doença de múltiplos órgãos causada por microdeleção de 25 genes no cromossomo 7 (q11.23), sugerindo uma vulnerabilidade ao estresse. Objetivamos determinar se crianças e adolescentes com síndrome de Williams-Beuren apresentam níveis elevados de estresse. Avaliamos 40 indivíduos em idade escolar, com diagnóstico de síndrome de Williams-Beuren e grupo controle. Os instrumentos utilizados: Escala de Estresse Infantil (ESI), Escala de Inteligência para Crianças (WISC), Escala de Inteligência para Adultos (WAIS) e um questionário semiestruturado. No grupo com o SWB, 50\% tinham altos níveis de estresse em comparação com $28,6 \%$ no grupo controle, diferença altamente significativa estatisticamente $(p<0,001)$. De escola de inclusão, $40,7 \%$ apresentaram maior estresse; de escola especial, 69,2\% ( $p>0,140$ ). Indivíduos com síndrome de Williams mostram índice elevado de estresse. Este estudo destaca a necessidade de orientação sobre a síndrome a pais e gestão escolar, com foco na redução de possíveis fatores ambientais estressantes.
\end{abstract}

Palavras-chave: Síndrome de Williams-Beuren, stress, crianças e adolescentes.

\section{Stress in children and adolescents with williams-beuren syndrome in school age}

\begin{abstract}
Williams-Beuren syndrome is a multiorgan disease caused by microdeletion of 25 genes on chromosome 7 (q11.23), suggesting a vulnerability to stress. In this study we aim to determine whether children and adolescents with Williams-Beuren syndrome have high levels of stress. We studied 40 subjects of school age, with confirmed diagnosis of Williams-Beuren syndrome and control group. The instruments used: Child Stress Scale (ESI), Intelligence Scale for Children (WISC), Adult Intelligence Scale (WAIS), and a questionnaire semi-estructured. In the group with SWB, 50\% had high levels of stress compared with $28.6 \%$ in the control group, statistically highly significant difference $(p<0.001)$. In Inclusion school, $40.7 \%$ revealed higher stress; special school $69.2 \%(p>0,140)$ difference was not statistically significant. Individuals with Williams syndrome show high level of stress. This study highlights the need for guidance about the syndrome to parents and school management, with focus on reducing of possible environmental stressors factors.
\end{abstract}

Keywords: Williams-Beuren syndrome, stress, children and adolescents.

\section{Estrés en niños y adolescentes con síndrome de Williams-Beuren en edad escolar}

\section{Resumen}

El Síndrome de Williams-Beuren es una enfermedad de múltiplos órganos causada por microdeleción de 25 genes en el cromosoma 7 (q11.23) sugiriendo una vulnerabilidad al estrés. El objetivo del estudio fue determinar si niños y adolescentes con síndrome de Williams-Beuren presentan niveles elevados de estrés. Evaluamos 40 individuos en edad escolar con diagnóstico de síndrome de Williams-Beuren y grupo control. Los instrumentos utilizados: Escala de Estrés Infantil (ESI), Escala de Inteligencia para niños (WISC), Escala de Inteligencia para Adultos (WAIS) y un cuestionario semi-estructurado. En el grupo con el SWB 50\% tenían altos niveles de estrés en comparación con 28,6\% en el grupo control, diferencia altamente significativa estadísticamente $(p<0,001)$. De la escuela de inclusión $40,7 \%$ presentaron mayor estrés; de la escuela especial $69,2 \%$ ( $p>0,140$ ). Individuos con Síndrome de Williams muestran índice elevado de estrés. Este estudio destaca la necesidad de orientación sobre el síndrome a padres y gestión escolar con enfoque en la reducción de posibles factores ambientales estresantes.

Palabras clave: Síndrome de Willians-Beuren, stress, niños y adolescentes. 


\section{Introdução}

A Síndrome de Williams-Beuren (SWB) é uma anomalia congênita rara causada por uma microdeleção hemizigótica do braço longo do cromossomo 7, banda 11.23, incluindo 25 a 35 genes (Bayés, Magano, Rivera, Flores, \& Perez Jurado, 2003; Merla, Ucla, Guipponi, \& Reymond, 2002). Pode apresentar alterações cardiovasculares, principalmente estenose aórtica supravalvar, estenose aórtica pulmonar e hipertensão (Pober, Johnson, \& Urban, 2008; Sugayama, Koch, Furusawa, Leone, \& Kim, 2004; Tassabehji \& Urban, 2006;), além de anormalidades renais e alterações no trato urinário (Sammour, Gomes, Duarte, Trigo-Rocha, \& Srougui, 2006), e anormalidades endocrinológicas, as quais incluem problemas na tireóide (Selicorni e cols., 2006). A incidência da síndrome é estimada entre 1:8000 e 1:20.000 nascidos vivos (Collins, Kaplan, Somes, \& Roma, 2010) e prevalência de 1:7.500 (Stromme, Bjornstad, \& Ramstad, 2002).

Estudos sobre fenótipo cognitivo, comportamental, social e de linguagem em pessoas com a síndrome descrevem padrões consistentes de deficiência intelectual em níveis variados, comportamentos elevados de sociabilidade e empatia, bom desempenho em tarefas que demandam uso de linguagem expressiva, prejuízos em habilidades de linguagem receptiva (Rossi, Moretti-Ferreira, \& Giacheti, 2007; Tassabehji \& Donnai, 2006), hiperacusia, comportamentos estereotipados, agressividade, comorbidades psiquiátricas como fobias específicas, transtorno de ansiedade generalizada, transtorno de déficit de atenção e hiperatividade (Gothelf, Farber, Raveh, Apter, \& Attias, 2006; Levitin, Cole, Lincoln, \& Bellugi, 2005; Leyfer, Woodruff-Borden, \& Mervis, 2009; Meyer-Lindenberg e cols., 2005; Nunes, 2010; Pober, 2010).

Esse perfil sugere que pessoas com SWB se expressam das mais variadas formas, com limitações que se confrontam entre a patologia e o que se espera de um padrão de normalidade provido da sociedade. Essa demanda de adequações provavelmente interferirá no desenvolvimento de habilidades adequadas de comunicação, socialização, realização de atividades da vida diária, desempenho acadêmico, dentre outras (Fish \& Nance, 2007; Vicari, Bellucci, \& Carlesimo, 2001). Pressupõe-se que exigências desajustadas, como padrões de comportamento, de argúcia e de aptidão contraditórias ao que se espera da deficiência intelectual, possam aumentar a vulnerabilidade de pessoas com SWB a reações de estresse, por suas condições clínicas.

Selye (1973) conceituou estresse como "qualquer adaptação requerida à pessoa" ${ }^{20}$. Estresse é definido por Lipp, Arantes, Buriti e Witzig (2003, p. 51) como:

[...] uma reação do organismo, com componentes físicos e/ou psicológicos, causada pelas alterações psicofisiológicas que ocorrem quando a pessoa se confronta com uma situação que, de um modo ou de outro, a irrite, amedronte, excite ou confunda, ou mesmo que a faça imensamente feliz.

A literatura descreve diversos fatores associados ao estresse. São fatores externos iluminação inadequada, excesso de barulho, dificuldades na interação social (discriminação) , falta de estimulação para o desenvolvimento de outras habilidades etc. Dentre os fatores internos, são citados pensamentos e ideias do próprio indivíduo em relação ao meio em que se encontra. Conforme as habilidades e os repertórios comportamentais da pessoa para enfrentar e manejar esses agentes, o nível de estresse variará.

Selye (1956, p. 128-139) estudou o excesso de hormônios pituitário e adrenal no organismo estressado e constatou consequências da influência nociva de agentes estressores, como doenças renais e cardiovasculares.

O eixo hipotalâmico-pituitário-adrenal (HPA) age como mediador das respostas adaptativas aos estímulos potencialmente estressores à medida que o indivíduo se prepara para lidar com a fonte de estresse (Fuchs e cols., 2001). Quando esse eixo se apresenta disfuncional, podem ocorrer elevações crônicas e persistentes de cortisol, provocando agudizações nas alterações cardíacas, pulmonares e renais, agravando o quadro clínico das crianças e adolescentes com SWB e submetendo-as ao risco de morte súbita, que muitos autores já apresentam como relacionada à própria síndrome (Wessel, Motz, Pankau, \& Bursch, 1997).

Tendo em vista a gravidade de muitas dessas reações fisiológicas para o desenvolvimento de crianças e adolescentes com SWB, o presente estudo tem como objetivo verificar se crianças e adolescentes com SWB que frequentam escolas de inclusão e escolas especiais apresentam índices elevados de estresse, de maneira a oferecer orientações para adequado ambiente escolar e melhor qualidade de vida.

\section{Método}

Os dados para a amostra foram coletados em três momentos: primeiro em entrevista com as famílias das crianças e adolescentes com SWB, durante a qual preencheram o questionário de caracterização social e demográfica. O segundo encontro destinou-se à avaliação de inteligência das crianças e adolescentes. E o terceiro encontro foi dedicado à aplicação da escala ESI de estresse. Os pacientes foram contactados por telefone para o agendamento dos encontros e foram atendidos individualmente em sala ambulatorial do Instituto da Criança do HCFMUSP. Em seguida, os responsáveis eram ouvidos para constatar a veracidade das respostas.

Por se tratar de amostras independentes, foram utilizados para tratamento dos dados a mediana e o teste não paramétrico de Mann Whitney.

Este estudo representa a análise do tipo observacional de 21 indivíduos do sexo masculino e 19 do feminino com SWB, todos com idade entre 7 e 18 anos, com diagnósticos clínicos confirmados e atendidos pelo HC. O grupo, a convite da Associação Brasileira da Síndrome de Williams e do Departamento de Genética do Instituto da Criança do HC, foi composto por moradores de diversos bairros da cidade e da grande São Paulo, bem como de outros Estados do território brasileiro. 
O critério de inclusão foi a confirmação do diagnóstico da SWB através de exames FISH, Molecular ou MLPA, constando a microdeleção hemizigótica no cromossomo 7 (q11.23), ter idade entre 7 a 18 anos e estarem matriculados no ensino fundamental ou escola especial. Todos os pais dos pacientes foram submetidos à entrevista inicial, assinaram termo de consentimento para a realização da pesquisa e responderam a um questionário de perguntas padronizado. Com o questionário, pretendia-se uma caracterização social e demográfica da amostra e, com base no Manual Diagnóstico e Estatístico de Transtornos Mentais (DSM-IV-TR) de 1995, apontar possíveis eventos traumáticos aos quais os participantes poderiam ter sido expostos nos últimos seis meses.

O instrumento de coleta de dados utilizado foi a Escala de Stress Infantil (ESI) para crianças e adolescentes. O ESI é um instrumento padronizado, brasileiro, validado para utilização de pesquisa e de fácil aplicação, que permite diagnosticar se a criança apresenta um quadro sintomatológico de estresse. O ESI é de alta confiabilidade, apresentando um coeficiente Cronbach Alpha de 0,90, maior que o apresentado pelo instrumento Lifestress Inventary (de 0,86 ), considerado altamente confiável por vários estudos de mesma finalidade (Bramston \& Fogarty, 2000). O questionário é composto por 35 perguntas devidamente organizadas para detectar Reações Físicas (RF), Reações Psicológicas (RP), Reações Psicológicas com Componentes Depressivos (RPCD) e Reações Psicofisiológicas (RPF). A avaliação de Quociente de Inteligência (QI) foi realizada por psicóloga da equipe do Departamento de Genética do HC, coautora deste trabalho.

Em função do resultado de avaliação cognitiva apresentado por algumas crianças e adolescentes com SWB, optou-se pela realização de um pré-teste na intenção de confirmar a confiabilidade na aplicação do instrumento ESI. Foi aplicado o mesmo instrumento em 20 crianças e adolescentes com suspeita de rebaixamento cognitivo e em suas respectivas mães respondendo sobre seus filhos, em momentos diferentes, totalizando 40 escalas. As respostas foram comparadas. Todos os participantes do pré-teste são atendidos na Secretaria dos Direitos da Pessoa com Deficiência, situada em Barueri, município da grande São Paulo.

A pesquisa foi aprovada pela Comissão De Ética Para Análise de Projetos de Pesquisa (CAPPesq), Número 944/08, do Hospital das Clínicas (HC) da Faculdade de Medicina da Universidade de São Paulo (FMUSP).

\section{Resultados}

Os resultados do pré-teste apontaram respostas semelhantes entre filhos e mães, que podem indicar confiabilidade nas respostas dos filhos. A mediana para filhos foi de: 35, mínimo: 12 e máxima: 74; mediana para as mães: 30, mínima: 6 e máxima: 64 - Teste Mann Withney: $p=0,212$. Não houve diferença estatisticamente significante. A maioria das respostas apresentou pontuação maior dos filhos, o que pode indicar que questões as quais remetem a percepções internas do indivíduo passam despercebidas pelas mães, talvez pela ausência de queixas. Todos os resultados de níveis de estresse foram concordantes entre os dois grupos (filhos e mães).

A amostra analisada no presente estudo apresentou idade média de 12 anos, mínima 7 e máxima $18(\mathrm{DP}=3,26)$. Foi constatada em todos os participantes a prevalência de deficiência mental, de acordo com resultados obtidos nos testes de inteligência com Escala de Inteligência para Crianças de Wechsler (WISC-III) e Escala de Inteligência para adultos de Wechsler (WAIS-III). Verificou-se que a média de Quociente de Inteligência Total (QIT) do grupo foi 68,5 (Desvio padrão=8,89) - compatível com deficiência mental leve a moderada.

A análise de estresse em relação ao sexo entre os grupos SWB e GC mostra que tanto meninos quanto meninas com SWB têm mais estresse que meninos e meninas sem a síndrome $(p<0,001)$, conforme tabela 1 e figura 1 .

Tabela 1. Estresse e Gênero na SWB e na GC

\begin{tabular}{lllllllllll}
\hline Sexo & \multicolumn{4}{c}{ SWB } & \multicolumn{5}{c}{ GC } \\
\cline { 2 - 7 } & \multicolumn{1}{c}{$N$} & Mdn & Min & Max & $N$ & Mdn Min Max & MW \\
\hline Fem & 19 & 40 & 19 & 84 & 19 & 17 & 6 & 54 & $p<0,001$ \\
Masc & 21 & 34 & 23 & 66 & 21 & 25 & 8 & 45 & $p<0,001$ \\
\hline
\end{tabular}

Nota. Fem= Feminino; Masc= Masculino; GC= Grupo Controle; Mdn= Mediana; MW= Mann Whitney. Normal até 39,6, alerta até 59,5 , resistência até 79,4 e exaustão $>79,4$.

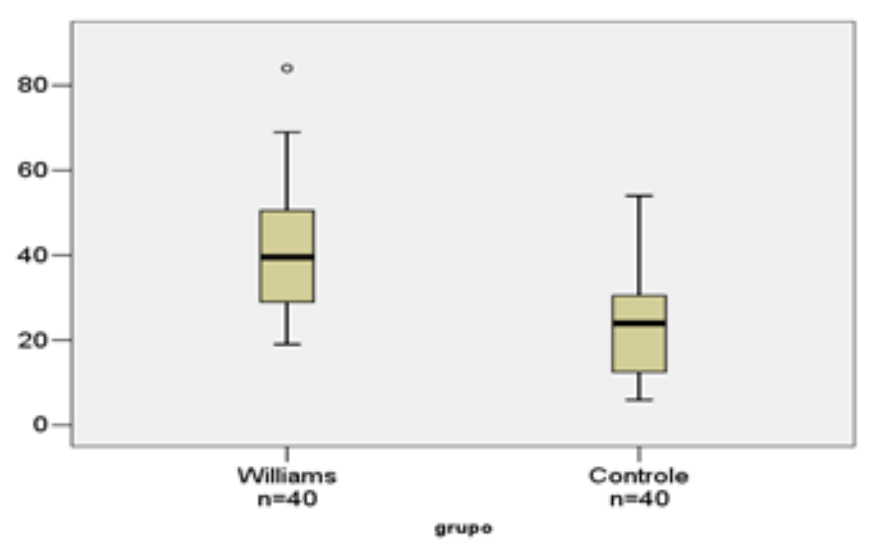

Figura 1. Comparação de estresse entre SWB e Grupo Controle

Em ambos os grupos, SWB e GC, os indivíduos com mais de 14 anos apresentaram níveis mais elevados de estresse (64\% e $45 \%$ respectivamente) em comparação aos grupos de menor idade ( $45 \%$ e $24 \%$ respectivamente).

A comparação do nível de estresse entre os grupos de indivíduos com SWB indica que meninas apresentam índice elevado de estresse, não houve diferença estatisticamente significante $(p=0,74)$, conforme tabela 2 . 
Tabela 2. Estresse e Gênero na SWB

\begin{tabular}{lccccc}
\hline & \multicolumn{5}{c}{ SWB } \\
\cline { 2 - 5 } & $\mathrm{N}$ & Mdn & Min & Max & \multirow{2}{*}{ tMW(p) } \\
\cline { 2 - 5 } Fem & 19 & 40 & 19 & 84 & \\
Masc & 21 & 34 & 23 & 66 & $\mathrm{p}=0,74$ \\
\hline
\end{tabular}

Nota. Normal até 39,6, alerta até 59,5, resistência até 79,4 e exaustão $>79,4$.

Tabela 3. Estresse Escolar na SWB

\begin{tabular}{lccccc}
\hline Escola & $\mathrm{N}$ & Mediana & Min & Max & tMann Witney $(\mathrm{p})$ \\
\hline Inclusão & 27 & 36 & 19 & 66 & \\
Especial & 13 & 50 & 21 & 84 & $\mathrm{p}=0,140$ \\
\hline
\end{tabular}

Nota. Normal até 39,6 , alerta até 59,5 , resistência até 79,4 e exaustão $>79,4$.

Tabela 4. Estresse e QI na SWB

\begin{tabular}{llllll}
\hline QI & N & Mediana & Min & Max & tMann Witney $(p)$ \\
\hline$<60$ & 18 & 41,5 & 23 & 62 & \\
$>60<89$ & 22 & 36,5 & 19 & 84 & $\mathrm{p}=0,935$ \\
\hline
\end{tabular}

Nota. Normal até 39,6, alerta até 59,5, resistência até 79,4 e exaustão $>79,4$.

Em relação ao ambiente escolar, o resultado mostra que não há diferença significante estatisticamente $(p=0,140)$ de estresse entre o grupo de crianças e adolescentes com SWB que frequenta escolas de inclusão em comparação com o que frequenta escolas especiais (ver tabela 3 ).

A amostra de crianças e adolescentes com SWB foi dividida em dois grupos considerando o nível de QI apresentado nos testes de inteligência: um primeiro grupo com QI abaixo de 60 e um segundo grupo com QI acima de 60 e abaixo de 89. O resultado indica maior estresse em crianças e adolescentes com QI menor de 60, diferença não significante estatisticamente $(p=0,935)$ conforme tabela 4 .

Foram analisados também os níveis de estresse em crianças e adolescentes com SWB em ambientes escolares específicos. Dos 27 indivíduos que frequentam escolas de inclusão, 16 (59\%) encontram-se em nível normal de estresse (mediana $=31), 8(30 \%)$ estão em estado de alerta (mediana $46)$ e $3(11 \%)$ em estado de resistência (mediana 62). Dos 13 que frequentam escola especial, $4(31 \%)$ encontram-se em nível normal de estresse (mediana 26), 5 (38\%) estão em estado de alerta (mediana 50), 3 (23\%) em estado de resistência (mediana 62) e 1 (8\%) apresenta estado crônico (nível de estresse 84). Vide tabela 5.

As respostas ao questionário aplicado para analisar a situação escolar das crianças e adolescentes com SWB revelam que mais da metade da amostra queixa-se de excesso de barulho e discriminação, $60 \%$ e $58 \%$ respectivamente, seguido de queixa de muita lição, 35\%, ver tabela 6 .

Tabela 6. Respostas do questionário sobre queixa escolar das crianças e adolescentes com SWB

\begin{tabular}{lcc}
\hline QUEIXA - N= 40 & N & $\%$ \\
\hline Não sabe ler & 5 & 12 \\
Não sabe escrever & 8 & 20 \\
Não entende a lição & 9 & 22 \\
Exigência da letra cursiva & 10 & 25 \\
Professor bravo & 8 & 20 \\
Muita lição & 14 & 35 \\
Muito barulho & 24 & 60 \\
Discriminação & 23 & 58 \\
Sente-se diferente dos outros & 10 & 25 \\
\hline
\end{tabular}

Tabela 5. Níveis de Estresse entre crianças e adolescentes com SWB em Escola de Inclusão e Escola Especial

\begin{tabular}{lcccccccc}
\hline Escola (n.) & Nor. & Mdn & AL (1DP) & Mdn & RES (2DP) & Mdn & EX (>2DP) & Mdn \\
\hline INCL (27) & $16(59 \%)$ & 31 & $8(30 \%)$ & 46 & $3(11 \%)$ & 62 & 0 \\
ESP (13) & $04(31 \%)$ & 26 & $5(38 \%)$ & 50 & $3(23 \%)$ & 62 & $1(8 \%)$ & 84 \\
\hline
\end{tabular}

Nota. INCL=Inclusão; ESP=Especial; NOR=Normal; AL= Alerta; RES=Resistência; EX=Exaustão; Mdn=Mediana. Normal até 39,6 , alerta até 59,5 , resistência até 79,4 e exaustão $>79,4$. 
A maioria dos pacientes da amostra não foi alfabetizada, apenas nove pacientes que frequentavam escola de inclusão foram alfabetizados, cujo QI variou em mínimo 51, máxima 77 e mediana 62. Alguns pacientes com QI total acima de 80 não conseguiram ser alfabetizados.

Os 14/40 pacientes que repetiram de ano, bem como os 13 que resistiam em ir para a escola apresentaram nível elevado de estresse, em média 43,4 e 47,7 respectivamente.

A leitura dos prontuários revelou que $87,5 \%$ da amostra apresenta personalidade expansiva e amigável; 95\% apresentam hiperacusia; 65\%, ansiedade; estenose aórtica supravalvar, 62,5\%; estenose aórtica pulmonar, $32,5 \%$; prolapso da válvula mitral, $25 \%$ e $17,5 \%$ apresentam hipertensão arterial. Foi constatado também que 2,5\% apresentam estenose da artéria renal e insuficiência renal, $30 \%$ apresentam enurese noturna e $27,5 \%$ têm alteração músculo-esquelética.

\section{Discussão}

O presente estudo confirma a presença de índice elevado de estresse nas crianças e adolescentes com SWB. Não foi encontrado na literatura nenhum estudo sobre estresse na SWB, apenas duas pesquisas (Gilmour, Skuse, \& Pembrey, 2001; Hessl e cols., 2002) utilizaram indicadores biológicos de estresse os quais avaliaram síndromes diferentes, tendo apenas deficiência intelectual como característica semelhante. O primeiro avaliou o nível de cortisol salivar em 109 crianças com síndrome de X-Frágil comparadas com 109 irmãos sem a síndrome e constatou um significativo aumento desse indicador hormonal que foi maior em meninos do que em meninas e ainda mais elevado em crianças com QI mais baixo. Outro estudo avaliou o estresse em pessoas com deficiência intelectual, analisado em 26 crianças com Síndrome de Prader Willi comparadas com 24 crianças com síndrome da baixa estatura e hiperfagia (HSS) e 20 crianças normais, utilizando os questionários Inventário de Comportamentos para Crianças e Adolescentes (CBCL) e Questionário para Professores (TRF) e medida de cortisol salivar. Os resultados indicaram níveis altos de cortisol no grupo de SPW, seguido do grupo HSS, seguido do grupo controle. A análise dos questionários mostrou a presença de diversos agentes estressores psicossociais no ambiente do qual participam as crianças com SPW e HSS. Esses resultados são concordantes com o presente estudo.

A personalidade amigável, a boa narrativa e o senso crítico presentes na população com SWB diferenciam-na de outras síndromes com deficiência intelectual. Os riscos de um otimismo desadaptativo, muito frequente na SWB, o qual pode ser irrealista, podem levar o indivíduo a lidar de maneira inadequada em certas circunstâncias, o que pode ter consequências desagradáveis que propiciem o estresse. Essa característica pode também facilitar a percepção da população com SWB em relação a estímulos estressores do meio, principalmente no caso dos adolescentes, devido a sua idade crítica, num período turbulento, cheio de mudanças que os tornam mais vulneráveis a perturbações (Goodman,, McEwen, Dolan, Schafer-Kalkhoff, \& Adler 2005; Romeo \& Mc-Ewen, 2006).

Crianças e adolescentes que frequentam escola especial são menos expostos a agentes estressores, entretanto, por terem maiores prejuízos em habilidades intelectuais e por ficarem menos expostos ao contato social mais amplo, acabam ficando mais vulneráveis a reações de estresse em ambientes diversos. Propor-lhes pequenos desafios e incentivá-los à independência são ações que podem contribuir para amenizar esse quadro.

O ambiente escolar per si dispõe dos mais variados agentes estressores, principalmente quando se trata de inclusão educacional se esta não se encontra eficazmente estruturada. Estando os alunos expostos a ambientes com excessivos estímulos sonoros, por exemplo, correm risco de terem a pressão arterial e o nível de cortisol alterado (Wälinder, Gunnarsson, Runeson, \& Smedje, 2007). Segundo dados dos prontuários clínicos, $85 \%$ da amostra apresenta hiperacusia, o que remete a um desconforto doloroso. As queixas mais frequentes foram: excesso de barulho, o que pode estar associado à presença de hiperacusia; discriminação, apesar do grau de deficiência intelectual, evidenciou-se que o senso crítico e a percepção do meio relacionados à habilidade de se comunicar estão presentes nessa população; e excesso de tarefas, o qual denota que a ação do professor diverge do conhecimento das características de déficit de atenção e déficit nas funções executivas nesta síndrome.

As alterações cardiovasculares e renais são características que podem ser indícios elegíveis para afirmar que indivíduos com SWB são mais vulneráveis ao estresse em ambientes diversos, principalmente no ambiente escolar. Considera-se que professores nervosos e impacientes, instruções confusas, tarefas em excesso, desvalorização das potencialidades dos alunos, competição demais entre os colegas e desrespeito às diferenças e às limitações se configuram em fortes fatores estressores externos e que têm uma grande participação no desenvolvimento do estresse infantil (Lipp \& Lucarelli, 1998), sendo, portanto, imprescindíveis orientações nesse sentido às instituições que atendem crianças e adolescentes com SWB.

Os resultados obtidos com este estudo mostram que crianças e adolescentes com SWB apresentam índices elevados de estresse. Não houve diferença estatisticamente significante entre o nível de estresse e gênero, tipo de escola e valor de $Q$ I nos pacientes com SWB.

Os tipos de reações de estresse mais acentuados foram Físicas e Psicológicas com Componentes Depressivos. As queixas mais frequentes de sintomas psicológicos neste estudo foram relativos a insegurança, desânimo e choro excessivo; de sintomas físicos, hiperatividade, dores de barriga e enurese noturna. Esses sintomas são oriundos de fontes externas, como qualquer evento do ambiente que exija adaptação - saber ler e escrever, barulho excessivo, independência nas atividades novas e de rotina etc., e de fontes internas, as quais se caracterizam pela forma como 
o indivíduo enfrenta situações novas ou de cotidiano, seus pensamentos e atitudes. Quando bem equilibradas, essas fontes proporcionam predisposição a mudanças, sensação de controle e motivação para envolvimento efetivo em alguma área da vida.

Foi observado tanto nas respostas dos questionários como nas escalas que crianças e adolescentes com SWB não têm recursos próprios para manejo de estratégias ou técnicas para lidar com estresse, seja por suas condições clínicas, seja por estarem em ambientes inadequados.

Segundo Lipp, Souza, Romano e Covolan (1991), as crianças adequadamente criadas são menos vulneráveis ao estresse e dele se recuperam com maior facilidade, pois seu poder de adaptação e seus recursos internos lhes são de valia nas horas críticas.

Em face à fragilidade orgânica de crianças e adolescentes com SWB, os achados apontam para a necessidade de capacitação de educadores e pais em relação à síndrome, capaz de incentivá-los a desenvolver ambientes controlados que permitam às crianças a aquisição de autonomia e independência, a estimulação de habilidades cognitivas e habilidades da vida diária.

Mostram-se importantes avaliações periódicas de estresse nessa população, seja através de escalas ou por medidas de cortisol, a fim de evitar o agravamento do quadro clínico.

Embora a amostra tenha sido pequena, os resultados deste estudo apontam índices elevados de estresse em crianças e adolescentes com SWB. Não foi encontrado na literatura nenhum estudo semelhante, o que dificultou comparações específicas. Sugere-se, portanto, pesquisas futuras neste formato.

\section{Referências}

Bayés, M., Magano, L. F., Rivera, N., Flores, R., \& Perez Jurado, L. A. (2003). Mutational mechanisms of Williams-Beuren syndrome e deletions. Am J Hum Genet, 73, 131-151.

Bramston, P., \& Fogarty, G. (2000). The assessment of emotional distress experienced by people with an intellectual disability: a study of different methodologies. Research in Developmental Disabilities, 21, 487-500.

Collins, R. T., Kaplan, P., Somes, G.W., \& Roma, J. J. (2010). Longterm outcomes of patients with cardiovascular abnormalities and Williams syndrome. Am J Cardiol, 105(6), 874-878.

Fish, G. S. \& Nance, W. (2007). Studies of age-correlated features of cognitive-behavioral development in children and adolescents with genetic disorders. Am J Med Genet A, 143A(20), 2478-2489.

Fuchs, E., Flügge, G., Ohl, F. e cols. (2001). Psychosocial stress, glucocorticoids, and structural alterations in the tree shrew hippocampus. Physiol Behav,73(3), 285-291.
Gilmour, J., Skuse, D., \& Pembrey, M. (2001). Hyperphagic short stature and Prader-Willi syndrome: a comparison of behavioural phenotypes, genotypes e indices of stress. British Journal of Psychiatry, 179, 129-137.

Goodman, E., McEwen, B. S., Dolan L. M., Schafer-Kalkhoff, T., \& Adler, N. E. (2005). Social disadvantage and adolescent stress. Journal of Adolescent Health, 37, 484-492.

Gothelf, D., Farber, N., Raveh, E., Apter, A., \& Attias, J. (2006). Hyperacusis in Williams Syndrome: Characteristics and associated neuroaudiologic abnormalities. Neurology, 66(3), 390-395.

Hessl, D., Glaser, B., Dyer-Friedman, J., Blasey, C., Hastie, T., Gunnar, M., \& Reiss, A. L. (2002). Cortisol and behavior in fragile X syndrome. Psychoneuroendocrinology, 27, 855-872.

Levitin, D. J., Cole, K., Lincoln, A., \& Bellugi, U. (2005). Aversion, awareness, and attraction: investigating claims of hyperacusis in the Williams syndrome phenotype. Journal of Child Psychology and Psychiatry, 46(5), 514-523.

Leyfer, O., Woodruff-Borden, J., \& Mervis, C. B. (2009). Anxiety Disorders in Children with Williams Syndrome, Their Mothers, and Their Siblings: Implications for the Etiology of Anxiety Disorders. J Neurodev Disord, 1(1), 4-14.

Lipp, M., Arantes, J. P., Buriti, M. S., \& Witzig, T. (2003). O estresse em escolares. Psicol. Esc. Educ. SP, 4(1), 51-56.

Lipp, M., \& Lucarelli, M.D. (1998). Escala de Stress Infantil - ESI. São Paulo: Casa do Psicólogo.

Lipp, M., Souza, E. A. P., Romano, A. S. F., \& Covolan, M. A. (1991). Como enfrentar o stress infantil. São Paulo: Editora Ícone.

Merla, G., Ucla, C., Guipponi, M., \& Reymond, A. (2002). Identification of additional transcript in the Williams-Beuren Syndrome critical region. Hum Genet, 110, 429-438.

Meyer-Lindenberg, A., Mervis, C. B., Sarpal, D., e cols. (2005). Functional, structural, and metabolic abnormalities of the hippocampal formation in Williams syndrome. J Clin Invest,115, 1888-1895.

Nunes, M. M. (2010). Avaliação do funcionamento cognitivo de pacientes com Síndrome de Williams-Beuren. Tese de Doutorado, Universidade de São Paulo, São Paulo.

Pober, B. R. (2010). Williams-Beuren syndrome. N Engl J Med, 362(3), 239-252.

Pober, B., Johnson, M., \& Urban, Z. (2008). Mechanisms and treatment of cardiovascular disease in Williams-Beuren syndrome. J. Clin. Invest, 118, 1606-1615. 
Romeo, R. D, \& Mc-Ewen, B. S. (2006). Stress and the adolescent brain. Ann NY Acad Sci., 1094, 202-214.

Rossi, N. F., Moretti-Ferreira, D., \& Giacheti, C. M. (2007). Perfil comunicativo de indivíduos com a síndrome de Williams-Beuren. Rev Soc Bras Fonoaudiol, 12(1), 1-9.

Sammour, Z. M., Gomes C. M., Duarte, R. J., Trigo-Rocha, F. E., \& Srougi, M. (2006). Voiding dysfunction and the Williams-Beuren syndrome: a clinical and urodynamic investigation. Urol.,175(4), 1472-1476.

Selicorni, A., Fratoni, A., Pavesi, M. A., Bottigelli, M., Arnaboldi, E., \& Milani, D. (2006). Thyroid anomalies in Williams syndrome: investigation of 95 patients. Am j Med Genet A, 140(10), 10981101.

Selye, H. (1956) The stress of life. New York: McGraw-Hill Book Company.

Selye, H. (1973). The evolution of the stress concept. Am Sci, 61, 692-699.

Stromme, P., Bjornstad, P. G., \& Ramstad, K. (2002). Prevalence estimation of Williams syndrome. J. Child Neurol, 17, 269-271.

Sugayama, S. M. M., Koch, V. H. K., Furusawa, E. A., Leone, C., \& Kim, C. A. (2004). Renal and urinary findings in 20 patients with Williams-Beuren syndrome diagnosed by fluorescence in situ hybridization (FISH). Rev. Hosp. Clin. Fac. Med. S. Paulo, 59(5), 266-272.
Tassabehji, M., \& Donnai, D. (2006). More or less? Segmental duplications and deletions in the Williams-Beuren syndrome region provide new insights into language development. European Journal of Human Genetics, 14, 507-508.

Tassabehji, M., \& Urban, Z. (2006) Congenital heart disease: Molecular diagnostic of supravalvular aortic stenosis. Methods Mol Med, 126, 129-156.

Vicari, S., Bellucci, S., \& Carlesimo, G.A. (2001). Procedural learning déficit in children with Williams syndrome. Neuropsychology, 7(39), 665-77.

Wälinder, R., Gunnarsson, K., Runeson, R., \& Smedje, G. (2007). Physiological and physiological stress reactions in relation to classroom noise. Scand J work Environ Health, 33(4), 260-266.

Wessel, A., Motz, R., Pankau, R., \& Bursch, J. H. (1997). Arterial hypertension and blood pressure profile in patients with WilliamsBeuren syndrome. Z Kardiol, 86, 251-257.

Recebido em: 23/01/2012

Reformulado em: 29/03/2012

$07 / 08 / 2012$

Aprovado em: 03/09/2012 


\section{Sobre os autores}

Vera Alice Alcantara dos Santos Amaral (veraalicesa@usp.br)

Faculdade de Medicina da Universidade de São Paulo, Psicopedagoga Mestranda do Programa de Pediatria

Endereço: Rua Vereador Derci Lourenço, 14 Itaquara Park - Pirapora - CEP 06550-000 SP.

Michele Moreira Nunes (michelemoreiranunes@yahoo.com.br)

Faculdade de Medicina da Universidade de São Paulo, Psicóloga, Hospital das Clínicas da Faculdade de Medicina da USP.

Endereço: Av. Dr. Enéas de Carvalho Aguiar - Cerqueira César - CEP 05403-900 - Sao Paulo, SP.

Rachel Sayuri Honjo (rachel.honjo@gmail.com)

Faculdade de Medicina da Universidade de São Paulo, Médica Geneticista, Instituto da Criança - FMUSP, Instituto da Criança - LIM36.

Endereço: R: Enéas Carvalho de Aguiar, 647 - Cerqueira César - CEP 05403-000 - Sao Paulo, SP

Roberta Lelis Dutra (lelis.roberta@gmail.com)

Faculdade de Medicina da Universidade de São Paulo, Médica Geneticista, Instituto da Criança - FMUSP, Instituto da Criança - LIM36.

Endereço: Rua: Enéas Carvalho de Aguiar, 647 - Cerqueira César - CEP 05403-000 - Sao Paulo, SP

Francisco Baptista Assumpção Jr (cassiterides@usp.br)

Instituto de Psicologia da Universidade de São Paulo, Médico Psiquiatra, Universidade de São Paulo, Instituto de Psicologia, Departamento de Psicologia Clínica.

Endereço: Av. Prof. Melo Morais, 1721 - Cidade Universitária - CEP 05508030 - São Paulo, SP

Chong Ae Kim (chong.kim@icr.usp.br)

Faculdade de Medicina da Universidade de São Paulo, Médica Pediatra, Instituto da Criança - FMUSP, Instituto da Criança - LIM36

Endereço: Rua: Dr. Enéas de Carvalho Aguiar nº 647 - Cerqueira Cesar CEP 05403-900 SP 